\title{
Investigating the Relationship Between Imagery and Rationale in Design
}

\author{
Shahtab Wahid, Stacy M. Branham, D. Scott McCrickard, Steve Harrison \\ Center for Human-Computer Interaction and Department of Computer Science \\ Virginia Tech \\ Blacksburg, VA 24060 \\ \{swahid, sbranham, mccricks, srh\}@cs.vt.edu
}

\begin{abstract}
Artifacts can be used to inspire, guide, and create new designs. As approaches to design can range from focusing on inspiration to formalized reasoning, we seek to create and study artifacts that combine the use of images and rationale. In this paper, we contribute an understanding of the relationship between imagery and rationale through an investigation of an artifact made of both. Through a study of group design sessions, we find images can provide access to rationale, moments of inspiration can be balanced with rationale, and differences between images and rationale must be reconciled. We conclude with thoughts on how such artifacts might be leveraged by the design community.
\end{abstract}

\section{Author Keywords}

Design artifact, imagery, rationale

\section{ACM Classification Keywords}

H5.2. Information Interfaces and Presentation: User Interfaces-Theory and Methods, Evaluation/Methodology

\section{INTRODUCTION}

Design artifacts are representations that express characteristics such as the properties of the object itself or captured information such as design constraints. During early design phases such as brainstorming, it is common for practitioners to leverage such artifacts to help inspire, guide, and create new designs. Research into design rationale explored ways in which knowledge about design could help in a design process (e.g., [15][5][3]), but many of these methods were shown to have time and effort costs that are not matched by their design benefits [10]. Our intention is to explore artifact presentation primarily focused on images rather than textual rationale, toward supporting the creation of early prototypes in design teams.

Permission to make digital or hard copies of all or part of this work for personal or classroom use is granted without fee provided that copies are not made or distributed for profit or commercial advantage and that copies bear this notice and the full citation on the first page. To copy otherwise, to republish, to post on servers or to redistribute to lists, requires prior specific permission and/or a fee.

DIS 2010, August 16-20, 2010, Aarhus Denmark

Copyright (C) 2010 ACM ISBN 978-1-4503-0103-9, 2010/08 - \$10.00.
Prior research explores how design knowledge can be captured in the form of reusable artifacts and stored in repositories for reuse [2][13][24]. These artifacts can often capture ideas in the form of rationale for specific design domains such as web design or social computing. Repositories in industry, one example being the Yahoo! Patterns Library [30], serve to share and distribute a common set of ideas that are collected over time. While experts can make use of the ideas contained within the rationale effectively and efficiently, in the forms of patterns or other repository-based methods, those who are unfamiliar with the design method or domain have a steep and costly learning curve. Our belief is that an imagecentric presentation method for rationale might be more accessible to a wide range of design teams-especially the novice designers we are targeting in our efforts.

While images can trigger the imagination of designers [17], we aim to also provide the opportunity to supplement the ideation that takes place. Our research trajectory has focused on seeking ways to encourage design teams to consider images first, inspiring designers to contribute their own interpretations before integrating the rationale [28]. We wish to promote inspired thought while also providing access to rationale to expand designer knowledge. We build on our previous work in which we created our own artifact set, in the form of cards, that make use of inspirational imagery and design rationale while targeting a specific design domain [28].

Our goal with this paper is to contribute an understanding of the nature of the relationship between imagery and rationale and identify the situations in which this relationship is made apparent. We present a study in which seven design sessions utilizing a set of artifacts developed by us yield insights into how both imagery and rationale, in the form of claims, function together. We then discuss how this approach might be viewed by those involved in the design community.

\section{RELATED WORK}

This work seeks to explore the effects of design artifact presentation on design teams, a widely explored area from the perspectives of design rationale and creative design. Methods leveraging design rationale built on prior lessons 
and justification for design decisions help create a new design firmly built on well-supported prior work. While rationale has appeared in many forms, a common form is the pattern, units of design recording contexts of use, conflicting forces, and potential solutions. Within HCI, Borchers [2] introduced the need for the encapsulation of the designer experiences through patterns, and Landay and Borriello [13] created patterns for ubiquitous computing to introduce lessons to new designers. Claims, a more lightweight design rationale artifact used in this paper, encapsulate the positive and negative tradeoffs of features and have been advocated as knowledge structures that can be stored in repositories and reused by designers [24]. The use of patterns, pre-patterns, and claims have shown promise in the design of interfaces [14][21][4]. However, many design rationale methods have been criticized as overly difficult or time-consuming in many situations, including for diverse teams in the early stages of design [10][7][22]. This work seeks to balance the presentation of rationale with the use of an image-first approach toward creating a prototype.

There are many examples of artifact representations that use images to inspire design. The Creative Whack Pack [27] and the Thinkpak [18] represent two of many popular creativity inspiring card sets. Approaches from industry and academia, like IDEO's Method Cards [12] and Friedman's Envisioning Cards [20] focusing on key design concerns, are particularly well suited for the design of novel interfaces and can be quite beneficial to novice designers. Herring et al. presented work on the use of examples-images, figures, and other things taken from information sources-during the design process, analyzing the use of these artifacts in the phases of preparation, idea generation, and idea evaluation [9]. These artifacts leverage imagery, augmented with questions and descriptions, to promote discussion among design teams through the inspiration provided by the images and text. However, none of these approaches explores the balance between artifacts as images and the associated rationale supporting them. The analysis of the tight coupling of image and rationale-balancing the rapid appeal of images with the in-depth knowledge of rationale-is the focus of this work.

The efforts in this paper focus on design in a shared workspace, an environment in which visual information about relevant shared objects is provided [29]. Investigations of shared workspaces for design sessions have also been carried out [6][25][19]. Tang and Liefer articulate the role of storing information, sharing ideas, and engaging attention with respect to the use of gestures [25]. Minneman's work serves to emphasize that design sessions are not just a set of technical processes, but that collaborative factors are intertwined [19]. While exploring the relationship between imagery and rationale, we also aim to provide examples of how the relationship is encapsulated within certain actions taken by the design team.

\section{INVESTIGATING IMAGERY AND RATIONALE USE}

Toward understanding the use of pictures and design rationale, we conducted a study of novice designers in which they were asked to construct systems for a given problem using our artifact set. Previous work in this line of research investigated how such artifacts facilitate the reuse of design knowledge [28]. First, the investigations pointed out that prototyping activities should be used to facilitate reuse. Second, it concluded that learning about the design domain and the artifacts themselves should be encouraged while making new design knowledge contributions for others to reuse. Third, it advocated that the structure of the artifact has implications for reuse [28]. In this paper, the artifacts are dissected to uncover the connections and tensions between the use of imagery and rationale and how it impacts the design process.

Because there is a need to represent the context and details of situations for a system being built [1], a design representation is a desired outcome when using our artifact set. Because of our emphasis on imagery, we asked participants to construct storyboard-like representations of their system using the artifacts we presented them with. Storyboards are visual narratives that function as early prototypes [26].

\section{Participants}

Twenty-one graduate students were gathered to take part in the study. All the participants were actively engaged in conducting HCI research or enrolled in a graduate HCI course at the time of the study. Their familiarity with storyboarding and claims varied. Due to our interest in looking for ways to provide new designers with rationale, we specifically preferred to recruit graduate students for this study because they are a pool of novice designers with initial academic training in design.

\section{Imagery, Rationale, and Other Materials}

As our goal was to investigate the presentation of rationale through imagery, we wish to describe the nature of the artifacts we created. We previously developed a set of cards that would combine the two concepts of interest together. Thirty cards relating to various features of notification systems, tools designed to make users aware of monitored information [16], were created. The features were chosen from a set of claims, rationale encapsulating the positive and negative tradeoffs of features [3], previously developed for notification systems [6].

The front of each card had a picture representing a feature along with a label to describe it. We searched online for creative commons licensed images that would be suitable for each card. As shown in Figure 1, the images were chosen such that a key characteristic of the feature could be emphasized immediately while also providing other information that could inspire different thoughts. For example, our card on the use of sliding transitions for notification was represented through an image of a news ticker on a building. In other cases, where the key item of 
interest was hard to isolate in a single picture, the appropriate area of the picture was highlighted with a circle.

The back of each card had a claim that came from the previously developed set for notification systems. Delivered in informal natural language, the claims address a variety of situational and interface aspects that affect the compatibility of the design and user models, such as user satisfaction and feeling of reward, color and object layout, and strength of affordances. The claim for the sliding transitions card in Figure 1 illustrates under what circumstances the feature might be appropriate for a given design situation.

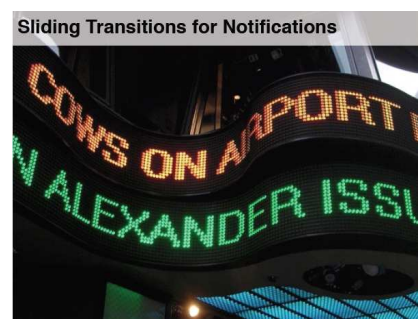

A sliding effect is used to transition from one notification to another

+ Can divert the attention of the user to the notification if needed

+ Can serve as a link between information pieces, allowing users to infer relationships between information pieces

- Constant use of sliding transitions can interrupt the user often

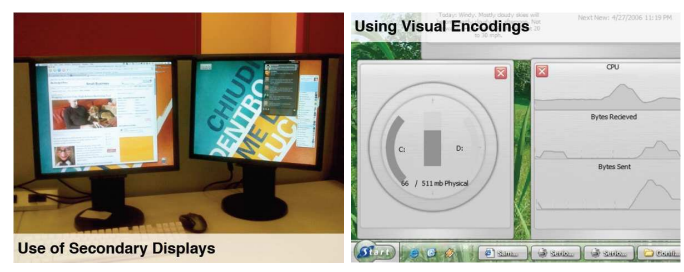

Figure 1. The front of the cards had pictures illustrating the design feature along with labels (top left). The back described the consequences of using the feature in a design (top right). Additional examples of cards are shown below.

The design sessions using our cards were carried out in a closed office that was setup with a table in the center and three chairs around it. The cards were scattered on the table upon arrival of the participants. Blank pieces of paper and pens were also provided so that new cards could be created if desired by the participants. A video camera was mounted on a desk nearby such that the whole table could be recorded. Two additional chairs were placed in the room for the investigators to observe the activity on the table.

Instructions for the task, design problem, and descriptions of claims and storyboards were provided. Each group was given a unique design problem that revolved around the need for a notification system for purposes such as notifying passengers in airports of flight status changes, nuclear plant operators of changing core temperatures, commuters of empty parking lot spots while driving, and theme park visitors of ride wait times.

\section{Procedure}

The participants were randomly divided into 7 groups of 3 people. Each group was asked to create a storyboard with 47 panels representing a system that would address the given design problem. A different design problem was provided for every design session. They were also given the option to create their own cards using the blank pieces of paper if they needed a feature that could not be found. Upon completion of the storyboard, they were asked to write a narrative for the storyboard describing a usage scenario. While reviewing the instructions, the participants were free to look at the cards and ask the investigators questions regarding the task. Once they read and understood all the instructions, they were permitted to start the design task. Each group was told they had 40 minutes, but we did not stop groups that went over the time limit.

Two investigators were present throughout each study session. Both investigators took notes about the actions and things said by the participants. Once the activity started, they only answered questions that related to the instructions. Any other questions were left up to the participants to resolve using their own judgment.

\section{Analysis}

The video recordings of all the study sessions were shared among the investigators. We took a grounded theory approach [23] to analyze the data. We adopted the open coding technique [23] in which we identified categories that we began to observe upon close examination of the videos. Categories of analysis included card familiarization, manipulations, comparisons, decision-making, and storyboard structure. Timing data was also collected during the analysis. Each group video was analyzed by two coders who watched the complete videos and identified critical points of interest based on the categories they developed.

\section{RESULTS}

In this section we present the observations we made in our investigation. We first discuss how we organize our results into phases of design activity. For each phase, we provide an overview of the range of observed activity. We then delve deeper into each phase and present examples of occurrences that highlight the relationships between imagery and rationale and the key actions that encapsulated the relationships.

\section{Phases of Artifact Use}

Studying the flow of our artifact, or card, usage illustrates to us that there are important processes that take place. Like Tang and Liefer [25] and Herring et al. [9], we prefer to first understand the structure of the activity to help guide our thinking. Since we took a grounded theory approach to the analysis, our initial analyses of the design sessions lead to the emergence of distinct phases. Our thinking suggests that the designers progressed through three phases: exploration, differentiation, and construction.

Exploration is marked by a state where designers begin to grasp the design task ahead of them and focus on acquainting themselves with the cards. During differentiation, the designers acknowledge the need for decision-making and begin to make choices as to what cards should be used. Construction marks the beginning of the assembling of chosen cards to form a system 
representation, illustrating how artifacts will connect to each other. During this time, the need for new artifacts with imagery and rationale might be identified. We proceed to use these three phases to organize the presentation of the results of our investigation.

\section{Phase Overview}

We first provide an overview of the range of activity we observed in each phase with respect to the use of the artifacts. In the exploration phase, groups spent 5 minutes and 7 seconds, or $11 \%$ of their time on average. Group 4 spent just 50 seconds in the phase while group 3 spent 7 minutes and 46 seconds. The participants spent time familiarizing themselves with the scattered cards in various ways. We counted activity such as touching a card or reading the label out loud as ways of familiarizing themselves with the cards and sharing cards. Looking at the total number of times a card was explored, we found on average 86 card explorations took place, ranging from 56 by group 5 to 102 by group 3. During exploration, a common act was to flip the cards over to read the claim. On average, the groups turned the cards over 20.7 times. Group 4 flipped cards the most, 33 times, and group 1 flipped cards just 10 times.

When we began to observe the groups making decisions regarding the cards, we marked it as the start of the differentiation phase. On average the groups spent 12 minutes and 12 seconds, or $28 \%$ of the session grouping, comparing, and deciding-key activities in differentiation. Group 2 spent just 27 seconds in the phase while the upper bound was set by group 1 with 13 minutes and 55 seconds. We also observed the continuation of exploring activity in the differentiation phase for all the groups. For example, group 4 explored an additional 86 times in this phase.

The construction phase began when participants placed cards to begin to assemble their storyboard. An average of 25 minutes and 38 seconds, or $61 \%$ of the time was spent in this phase. Group 1 completed the phase in 13 minutes and 4 seconds while group 7 spent 56 minutes and 2 seconds. The final sizes of the storyboards varied a lot. Group 1 had a storyboard with 5 cards while group 5 had 14 cards. There was considerable shifting in the number of cards being placed in the storyboard. At one point the storyboard for group 3 had 13 cards in it. They eventually narrowed it down to 9 cards. Group 7 also had up to 17 cards before they finally settled on 12 . On average 12 cards were considered for inclusion in the storyboards, but at the end of the sessions, an average of 9 cards were included. Note that we had asked for the storyboards to have 4-7 panels, but an average of 9 cards were used because certain cards were grouped together within the same panel. During this phase we also observed new artifacts being created. Three design sessions, groups 1, 4, and 7, decided to create new cards for their storyboards. As with the differentiation phase, we also observed exploration and differentiation related activity in the construction phase, although this was less.

\section{Phase Activities}

In our analysis of each phase we investigated the role the cards played toward identifying and describing the nature of the connections between imagery and rationale. We then isolated the activities that most embodied these relationships. In the following description of the results, we present examples of events that best describe the relationships and their corresponding activities.

\section{Exploration}

The use of images to summarize the idea of the artifacts was critical to how the participants recognized and explored the cards. This was important because of the number of cards that were scattered across the table during the study sessions (see Figure 2). A participant in group 1 commented on the utility of the pictures: "For me it was the main thing. I first looked at the photo and then read it through." A participant in group 7 emphasized the utility of the pictures: "the picture...makes it easier to identify the object so it stands out from the other objects. The pictures are quite different so maybe we employ less effort to recognize the objects instead of going through the text." In response to this comment, a participant in the same group said, "if you had pieces of paper with text on them it would be harder to sort through them." Such comments point to two possible benefits of using imagery in this way. First, the imagery provides a method to represent the ideas that are trying to be conveyed through the rationale. Second, the image is the primary mechanism through which access is provided to rationale when necessary-especially in conditions where designers might have access to too much rationale and a limited capacity to go through all of it at once.

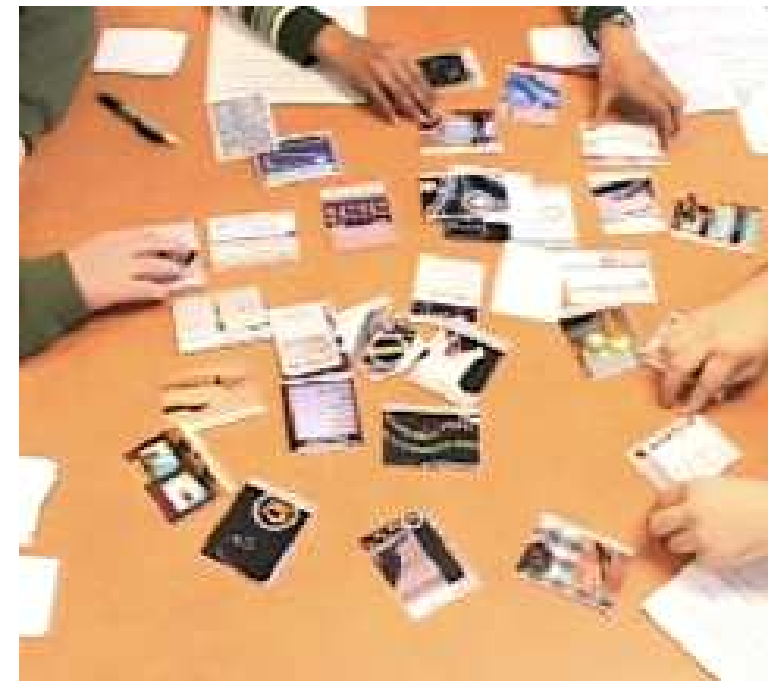

Figure 2. Collaborators from group 6 in the exploration phase. The cards remained scattered as group members familiarize themselves with the cards and make suggestions.

The imagery also presents a consequence that might be interpreted negatively by some. In some cases, there were instances where there was confusion regarding what a card was actually about due to the nature of the picture. Our 
sporadic notifications card was about the idea of notifications coming at unexpected times. Because representing this idea in the form of an image is not necessarily easy, we chose to use a picture of a phone to point out that incoming phone calls occur at irregular intervals. However, a group 6 participant referring to this card mentioned, "when I first see this I think phone, not sporadic notification. It seemed like the labels were a lot more useful than the actual screenshots." In this case, the picture failed to communicate what we as the designers of these cards intended to deliver. However, the label on the card served to point the participants in the direction we envisioned for the image. While this may seem like a negative effect, we also discuss later how this can also have a positive impact.

The claims served as a way for the participants to familiarize themselves with the details of an artifact. A group 3 participant mentioned, "sometimes pictures cause some confusion so we can go back to the claims to try to get more information to confirm our understanding." Also speaking of the claims, a group 4 participant said, "I guess it clarifies what the creator of these [cards] intended to mean by each of these." It served to clarify thoughts if designers were confused about the cards because they misinterpreted or were unsure of what a picture meant. In one instance we saw two participants in group 7 who were looking at a card about personalizing notifications and were unsure about what the feature was about. They flipped it over and one participant said, "this seems to be more about the mode of notifications rather than... which things you're being notified about...it's more about the mode than the content [of notifications]." In another example, a group 2 participant looked at a card about secondary displays and asked, "do we know what a secondary display is?" $\mathrm{He}$ flipped the card over to read the claim and then said, "I guess that works." Thus, in this initial exploration phase, it seems these examples illustrate that the claims do not serve as decision-making mechanisms, but rather as agents that inform designers about the artifacts at hand.

Within this phase, the key relationship between images and rationale is that of access. The examples mentioned tell us the use of imagery can potentially make it easier to find and read rationale that might have otherwise been harder to sift through. If there were no images at all it would potentially be harder to read through large amounts of text. Once accessed, the rationale serves to clarify the intended meaning of the artifacts and its potential uses for the designers.

This combination of methods to familiarize oneself with an artifact led to the most common activity that took place in this phase: suggesting. In fact, it is within the act of suggesting that we encounter members access rationale. To explain how this occurs, we provide examples of how cards might be suggested to others in the group. We were able to identify an average of 16.2 suggestions per group. Group 2 set the minimum at just 7 instances and group 1 the most with 23 cases. Participants would discover cards and offer them as potential solutions to the problem at hand. One common method was to read the card's label out loud for the rest of the group to hear and consider. The following example from group 4 demonstrates how one such suggestion can occur:

RB: [nudges then picks up a card, flips to read the back, places card centrally and makes a rigid pointing gesture]

AA: [picks up card and places it near SV]

SV: [picks up card and puts it in the 'keep' card pile]

This example demonstrates how a member selected a card, accessed its rationale, and then suggested the card to others in the group. Having understood the card was evaluated by a member, AA and SV both acknowledged this in their handling of the card, leading the card to be placed in a pile of potential picks for their system.

\section{Differentiation}

During this phase we observed the participants trying to assess the utility of the cards toward making decisions within the context of the given design problem (see Figure 3 ). The images facilitated creative thinking, allowing the designers to investigate how a card might be used. For example, one card about showing trends in data had a picture of a graph showing changes in values over time. Group 1 was told to create a system that would allow commuters to find empty parking lot spots. A participant from this group looked at the trends in data card and said, "you see [some spot] is empty so you click that one and it just opens up...[shows] when it got available and also this graph...you know...gives you an idea about what time of the day it gets full and what time of the day it gets [empty] and stuff like that. That might be a cool thing I think." This illustrates how images tied to the idea represented by the card can literally become the feature the designers choose to use in their own work.

In other assessment cases, an idea was extracted from a picture, but was unrelated to the feature the picture was representing - perhaps a more creative act. Previously we discussed misinterpretations of images as a potential negative consequence of the imagery. However, in other cases we found that ideas not related to the card, such as the sporadic notification card being interpreted as a card about a phone, can be beneficial to the designers. For example, a card about relating preexisting user knowledge to a notification had a picture of a chat window with a chat history. The history was meant to point out that the current chat message notification could be related to a previous chat that took place in the past. A participant from group 4 picked up the card and said "if you want a timestamp...then [use] something like this." The timestamps happened to be a part of the picture, but were not there to illustrate the idea of the card. However, in this case the timestamp was 
extracted as an element of interest to serve a different function in their design-demonstrating that the image can serve to jumpstart other ideas that may be beyond the scope of the card.

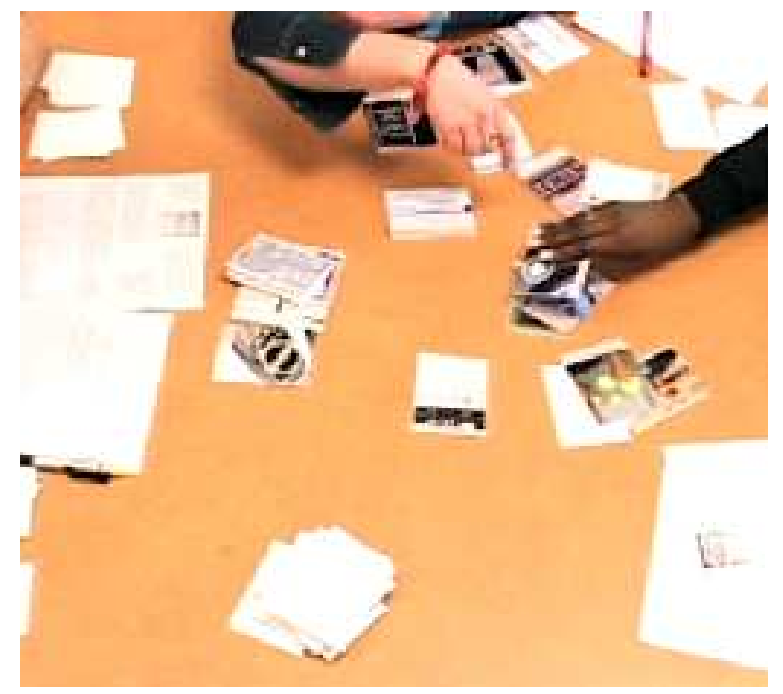

Figure 3. Group 5 in the differentiation phase. A group member places a card onto one of the five existing piles as decisions about their utility are being made. A few scattered cards are yet to be checked.

While in the previous phase the rationale served to familiarize the participants with the artifact, in this phase the participants used the claims to judge whether the artifact might be utilized in the design. For example, one common way of judging was to determine how general or specific the artifact is. The scope of the artifact mattered because it could potentially determine how applicable the artifact could be. We had one card that was about notification systems. Several groups identified this card as being a very general card with wide applicability. One person in group 2 mentioned, "[the] notification systems [card] seems too general for this task." This same kind of observation was made by another member of group 3. She pointed at several cards first and then pointed to another set as she said, "I think these are somewhat high level features and these are more into details."

Another common differentiating method was to compare alternatives to each other. In group 1, a member looking at the sporadic notification and continuous notification cards identified them as claims that are alternatives to each other. The person said, "sporadic vs. continuous...this is something we need to think about. Will it be continuously displayed on your handheld?" In another instance a person in group 1 looking at cards about voice and touch mention: "I just noticed this voice one before...I put it up next to the touch one. I guess we could...yeah maybe we could look at the pros and cons of each and see what fits better or maybe what downside will be more [tolerable]." The claims play a critical role in aiding the participants to identify what to use. The scope and the tradeoffs are taken into account because the designers want to make sure that the use of the card is feasible.

In this phase, the relationship between the images and the rationale change to that of balance. A more promising utility of images lies in their ability to inspire potential uses and new ideas, but these creative sparks might occur without careful thought. Referring to the rationale of the card, and even the rationale from alternative options, allows the designers to compare some of their initial thoughts to the potential consequences of using the card. Thus, creative thought fostered by the imagery is checked and balanced by the knowledge embedded in the rationale when assessing the utility of a card.

This balancing act was observed in the decision-making activity throughout the design sessions. As potential uses and alternative options arise, the designers need to take these into account when making a choice. This was most apparent when comparing cards to each other. On average 3.5 comparisons were observed in the groups. Group 7 had 1 instance while group 3 had the most with 7 comparisons. The following example from group 3 demonstrates how an explicit comparison took place:

RW: [places two cards, claim-up on the table in a central area]

"I think these..."

[points to cards, one with each finger, then retreats]

"two items are..."

SP: [touches the edge of one of the cards and pauses]

RW: $\quad$ "very similar..."

[points to cards with fingers]

"to each other."

At a time when a decision needed to be made, the member first might have thought that the two cards might be quite different from each other due to the different imagery-a result of her interpretation of the picture. However, upon further inspection her interpretation was checked by the claim on the card, leading her to conclude that the two cards are fundamentally similar.

\section{Construction}

In this phase the construction of the design representation, the storyboard, was initiated. Critical to this phase was the linking of cards together to create the storyboard (see Figure 4). These connections were often triggered by discussion about the images and the claims. Because the images served as creative springboards, the exact meaning of the images to suit the context of the design had to be agreed upon. For example, certain objects or ideas that existed within the images were emphasized as the source of the meaning. Recall the example with the use of the timestamp on the relating preexisting user knowledge to notification card and the spontaneous notification card with a picture of a phone. In both cases the source of the meaning was not related to the intention of the card. To 
avoid any discrepancies, these interpretations had to be agreed upon through discussion focused around the image and even the claim at times so that how the card is used in the system would be clear to everyone.

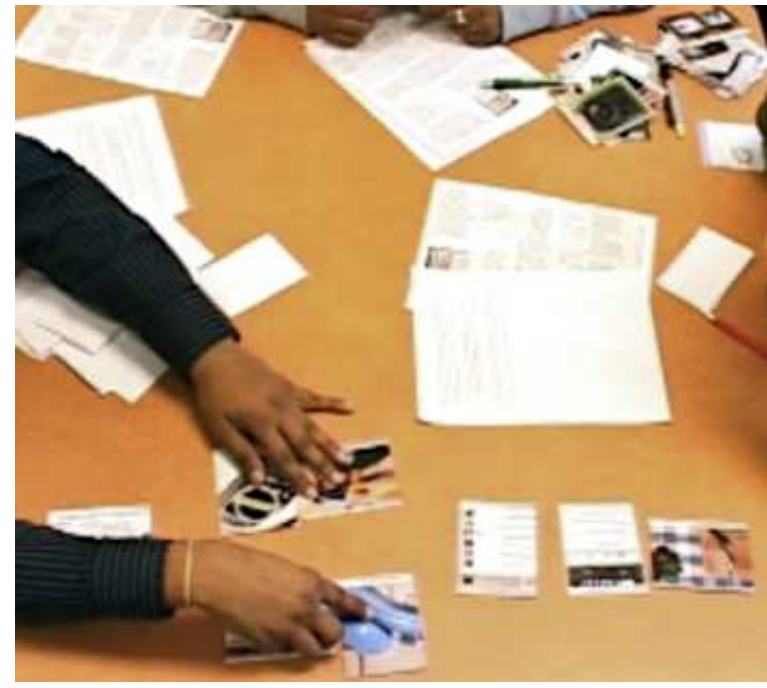

Figure 4. Group 4 in the construction phase. A group member sequences the cards within the storyboard.

This was especially apparent when new cards were created by 3 groups. A motivating factor for creating a new card was the absence of a feature that seemed to be necessary. This was usually realized when trying to link cards together to construct the system. When a new image was drawn for the new card, the aspects that were most meaningful to the group were emphasized in the picture-signifying agreement on what the image would mean for them. Group 1 created a card called using geospatial representation of information so that they could use it for their commuter parking lot system (see Figure 5). The group drew a picture of a simple road map to emphasize the geospatial capabilities of the artifact in relation to navigation. Another group later edited this same picture and added points of interest to the sketch, reemphasizing the key aspects of the image that mattered to them the most.

When the claim for this new card was written, the group considered the possible tradeoffs of using such a featuremaking sure to think of both positive and negative consequences. The member primarily writing the claim on the back of the card wanted to make sure the claim was well-connected to what the image was depicting-putting effort into reducing possible misinterpretations by others.

The act of linking cards to construct the storyboard was often also triggered by the claims. Connections between cards were created depending on certain needs established by the group. For example, if the participants identified a card that had a specific negative consequence that was unacceptable, they chose to look for another artifact that might mitigate the negative effects of the artifact. For example, a participant in group 3 who looked at a claim downside for a card said, "...may miss a notification and wait...but we can compensate it with this one. If they miss it they will get a continuous notification like an icon on the corner."

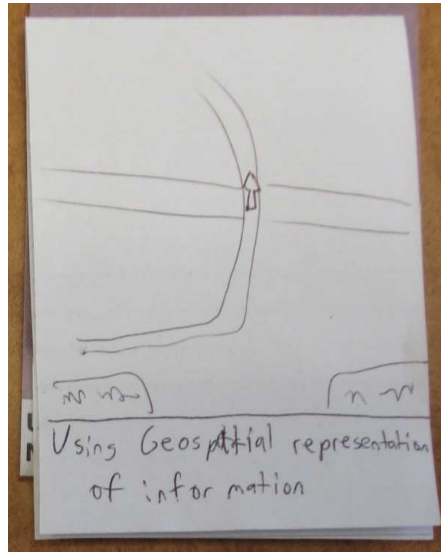

Figure 5. A new card with a picture and claim was created by group 1 when the needed arose while assembling the storyboard.

Often cards were combined together such that both cards would be used in conjunction to represent a new idea. For example, a new notification method could be created by combining a card about a blinking light with an audio notification card to represent a blinking light that would also provide audio feedback. Group 3 discussed joining a large-screen public display with a peripheral display to create a less distracting and user-driven source of information. While the claims for the two cards complement each other, they still remain as two distinct claims in the storyboard, but are interpreted by the group as one artifact. It is important to note that the claims have to be able to work together. It would not make sense for a claim about the use of gestures to necessarily be combined with the use of a handheld device.

The participants also linked cards appraising the state of an interface to cards about interacting with the elements in the interface. As the participant in group 1 who wanted to use the trends in data card mentioned, the use of the card was also tied to how a user would interact with the feature once included. Another typical example would be to link a card on the use of icons with a card on the use of a mouse to represent a certain task flow.

In the construction phase, the relationship between images and rationale revolves around the theme of reconciliation. The example of the timestamp in the picture of the relating notification to preexisting user knowledge card demonstrates how the members had to reconcile their reappropriation of the image with the claim for that card. In cases, where a card was being created, the members did not have to explicitly reconcile the drawn image and the claim, but efforts were put into ensuring that both were tightly connected so that others who choose to use that card would not use it differently. Finally, claims themselves must be reconciled with the images and claims of other cards. As in 
the example where a downside is unacceptable and needs to be resolved with another card, the members still need to judge whether the other image and claim might cause any discrepancies. If it is the case, then the other cards may not be linked together or it might mean that a new and different card is needed.

An activity core to the assembling of the storyboard itself is sequencing, in which the group tries to formulate the order of the cards, signifying not just what is used, but how it is used-a process that embodies reconciliation. Most groups went through several stages of the storyboard, repositioning cards and adding or removing a card whenever appropriate. On average, cards were added or removed 16 times. Group 1 did this just 9 times and group 2 did the most with 25 times. Often group members would create the storyboard piecewise. In the following group 3 example, a portion of the storyboard was identified:

AS: "So it's going to be a notification..."

[shifts 'sporadic notification' card up on the table]

"which..."

[shifts 'blinking light' card and 'textual notification'

cards into a second row beneath 'sporadic

notification' card]

"will be..."

[moves 'tactile notification' card into the second row with previous two cards]

"all three... going into..."

[shifts two more cards into rows three and four]

"something like this: Arrow..."

[gestures an arrow at upper third of the storyboard]

" arrow ..."

[gestures an arrow at middle of the storyboard] " arrow "

[gestures an arrow at bottom third of the storyboard]

SP: "Yeah."

RW: "Mm hm."

In the example, through discussion of the cards that could be in the storyboard, AS presents a sequence for the cards. While the combined use of a blinking light and a textual notification might seem odd, he reconciles their presence with the inclusion of the sporadic notification cardillustrating the light and textual notification are two forms of sporadic notifications occurring in the system.

The sequencing and reconciliation that took place was reflected in the final structure of the storyboards. Generally, the cards were laid out horizontally next to each other to indicate progression through the usage scenario (see Figure 6). When features were combined after reconciliation, the cards would be placed next to each other or overlapping slightly. Group 5, for example, had 5 panels that contained 2 or more cards within them and group 7 had 3 such panels. Groups 2, 4, and 6 had 2 panels each.

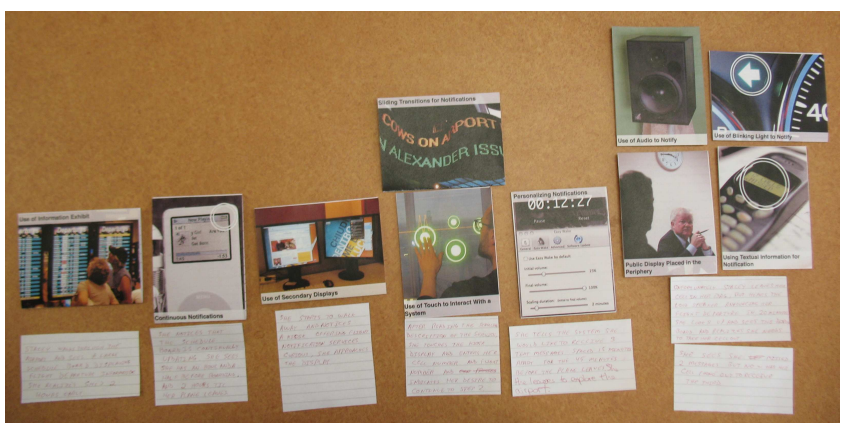

Figure 6. An example of a 6 panel sequence of cards created for a storyboard after reconciling differences within and across panels.

\section{DISCUSSION}

Our investigations using our card set led us to identify three types of relationships that exist between imagery and associated rationale: access, balance, and reconciliation. While we acknowledge our investigation was conducted with novice designers - our target group-we would still like to suggest some thoughts regarding how our cards and the identified relationships might be viewed by those in the design community since we have seen limited work on this so far.

The structure of our artifacts led us to explore how they might serve two different approaches to design: communities that prefer to focus more on idea generation through inspiration and communities that emphasize rationale as a central tenet. On one hand, communities that prefer techniques that focus on idea generation and inspiration have leveraged the use of imagery as a vital design tool [9]. Unlike other card decks such as the IDEO Method cards [12], Creative Whack Pack [27], and Thinkpak [18], we sought to connect closely the inspiration provided upon seeing the image with the utility through rationale of the images-emphasizing the need for both balance and reconciliation. For example, the image in an IDEO Method card with a woman holding a bird on one side and a description of rapid ethnography on the other provides little relevant design knowledge to most users, while in our card set our efforts to use tightly-connected domain-specific cards with pictures and labels on one side and elaborations of the pictures on the other enable us to better reflect key concepts through the use of imagery. This can even create a smaller gap between the interpretation of the image and the rationale. Through our study, we found that the images serve to not only inspire new ideas both literally and through reinterpretation, but also serve as references to concepts in the rationale-as exemplified by participant comments that the rationale "clarifies" and "suggests" rather than imposing or requiring, as would seem to be a danger in many rationale-centric design approaches.

On the other hand, a community that believes in the use of rationale as the central tenet to design would necessitate a 
tight inclusion of rationale. Our study revealed that the rationale plays a small but influential role in familiarizing the designers with the artifacts, assisting with decisionmaking, and creating new designs. For example, as noted in our study results, participants looked at the rationale on the back of the card an average of 20.7 times-fewer than once per card. While rationale is made up of the reasoning behind design ideas [2][3], the presentation of such rationale can make it hard to use [10]. Certainly our investigation found that we can provide access to rationale through images, but questions of whether this method-or something like it - provides and requires adequate access to the rationale for a given design project necessarily remain for the leader of a design team.

The unique manner of artifacts allowed us to explore the tradeoffs between these two approaches to designing interactive systems. A common theme that emerged from this is that ultimately we must focus on how the artifacts are understood and used with each other through connections established between them. This same notion can also be seen in Halskov and Dalsgard's Inspiration cards when they are ultimately chosen and combined together on a poster [8]. While we seek to support such a goal, we recognize that the actions taken by designers determine which relationships between images and rationale come into play. We find actions-such as suggesting, decision-making, and sequencing - serve to ultimately create this context. We do acknowledge that there may be other design actions that we may not have encountered.

As we build on the vision of Minneman [19], the results of this paper serve to illustrate the role of such artifacts within design - specifically those intended to inspire thoughtful design, whether in card sets, online tools, knowledge repositories, or methodologies. Knowledge of the relationship between imagery and rationale and their associated actions may aid the development of other artifacts and supporting tools.

Summarizing this work and looking toward future design, tool-building, and evaluation efforts, it seems important that rationale supporting an artifact be presented in a designerdigestible format. Of course, the nature of that format depends on a great many factors, e.g., the homogeneity of the design team, familiarity of the team with the problem, or importance to use and maintain design rationale. In a situation when ideation is of paramount importance, it may be most prudent to use purely image-based card decks that can be quickly understood at a surface level, but that may be richly examined by a diverse design team with different perspectives. A situation where it is essential to maintain rationale may require the design team to invest extra time to understand the claims, patterns, or other rationale mechanisms and content, but the extra effort may facilitate necessary verification and evaluation steps. We suspect that most situations lie in between these two extremesnecessitating further development and exploration of the types of image-based rationale presentation introduced in this paper.

\section{CONCLUSION}

This paper aimed to investigate the relationship between imagery and design rationale. We introduced a set of artifacts, cards, that contain both images and rationale. By observing design sessions that leveraged these cards, we identified the kinds of relationships that exist. We found the relationships revolve around providing access to rationale through imagery, balancing inspiration with rationale, and reconciling differences between images and rationale that might present themselves. We then discussed how our kind of artifact might serve design communities that rely on inspiration and communities that focus on rationale as a central theme.

\section{ACKNOWLEDGMENTS}

We thank the participants of our study and those who provided us with valuable feedback regarding the directions of this work.

\section{REFERENCES}

1. Bodker, S. Understanding representation in design. Human-Computer Interaction, 13, 2, pp.107-125, 1998.

2. Borchers, J. O. A Pattern Approach to Interaction design. In Proceedings of the conference on Designing Interactive Systems (DIS 2000), pp. 369-378, 2000.

3. Carroll, J. M., \& Kellogg, W. A.: Artifact as theorynexus: Hermeneutics meets theory-based design. In Proceedings of the SIGCHI Conference on Human Factors in Computing Systems (CHI 1989), pp. 7-14. 1989.

4. Chewar, C. M. and McCrickard, D. S. Links for a Human-Centered Science of Design: Integrated Design Knowledge Environments for a Software Development Process. In Proceedings of the 2005 Hawai'i International Conference on System Sciences (HICSS '05), 2005.

5. Conklin, J., and Begeman, M. L. gIBIS: a hypertext tool for exploratory policy discussion. ACM Transactions on Information Systems (TOIS), 6(4), pp. 303-331. 1988

6. Fabian, A., Wahid, S., Bhatia, S., and McCrickard, D. S. Creating an Interactive Learning Environment with Reusable HCI Knowledge. In Proceedings of the World Conference on Educational Multimedia/Hypermedia and Educational Telecommunications (ED-MEDIA '06), pp. 2314-2322, 2006

7. Grudin, J. Evaluating Opportunities for Design Capture. In T. Moran \& J. Carroll (Eds.), Design Rationale: concepts, techniques, and use, pp. 453-470, 1996.

8. Halskov, K. and Dalsgård, P. Inspiration card workshops. In Proceedings of the Conference on Designing interactive Systems (DIS 2006), pp. 2-11, 2006. 
9. Herring, S. R., Chang, C., Krantzler, J., and Bailey, B. P. 2009. Getting inspired!: understanding how and why examples are used in creative design practice. In Proceedings of the 27th international Conference on Human Factors in Computing Systems (CHI 2009), pp. 87-96. 2009.

10.Horner, J., and Atwood, M. E.: Design rationale: rationale and the barriers. In Proceedings of Nordic Conference on Human-Computer Interaction (NordiCHI 2006), pp. 341-350, 2006.

11. Hutchins, E. \& Klausen, T. Distributed cognition in an airline cockpit. In Cognition and Communication at Work. Cambridge University Press, 1996.

12. IDEO Method Cards. (2003) [S.I.]: IDEO.

13.Landay, J. A. and Borriello, G. Design Patterns for Ubiquitous Computing, Computer, 36, 8, pp. 93-95, 2003.

14.Lin, J. and Landay, J. A. Employing patterns and layers for early-stage design and prototyping of cross-device user interfaces. In Proceeding of the SIGCHI Conference on Human Factors in Computing Systems (CHI 2008), pp. 1313-1322, 2008.

15. MacLean, A., Young, R., Bellotti, V. and Moran, T. Questions, Options, and Criteria: Elements of Design Space Analysis. Human-Computer Interaction, 6 (3\&4), pp. 201-250. 1991.

16. McCrickard, D. S., Chewar, C. M., Somervell, J. P., and Ali Ndiwalana. A Model for Notification Systems Evaluation-Assessing User Goals for Multitasking Activity. ACM Transactions on Computer-Human Interaction (TOCHI), 10, 4, pp. 312-338, 2003.

17. McKim, R. H. Experiences in visual thinking. Brooks/Cole Publishing Company, 1972.

18. Michalko, M. Thinkertoys. Ten Speed Press, Berkeley, CA, 2006.

19. Minneman, S. L. The Social Construction of a Technical Reality: Empirical Studies of Group Engineering Design Practice, Xerox PARC Technical Report, SSL-91-22 (Ph.D. Dissertation, Stanford University), 1991.

20.Nathan, L., Friedman, B., and Hendry, D. 2009. SUSTAINABLY OURS: Information system design as catalyst: human action and environmental sustainability. interactions 16, 4 (Jul. 2009), pp. 6-11.

21.Saponas, T. S., Prabaker, M. K., Abowd, G. D., and Landay, J. A. The impact of pre-patterns on the design of digital home applications. In Proceedings of the Conference on Designing interactive Systems (DIS 2006), pp. 189-198, 2006.

22. Selvin, A. M. Supporting Collaborative Analysis and Design with Hypertext Functionality. Journal of Digital Information, Vol 1, No 4, 1999.

23. Strauss, A., and Corbin, J. Basics of qualitative research: Grounded theory procedures and techniques. Sage Publications, Inc., Newbury Park, CA, 1990.

24. Sutcliffe, A. G. and Carroll, J. M. Designing Claims for Reuse in Interactive Systems Design. International Journal of Human-Computer Studies, 50, 3, pp. 213 $241,2000$.

25.Tang, J. C. and Leifer, L. J. A framework for understanding the workspace activity of design teams. In Proceedings of the 1988 ACM Conference on Computer-Supported Cooperative Work (CSCW 1988), pp. 244-249, 1988.

26. Truong, K. N., Hayes, G. R., and Abowd, G. D. Storyboarding: an empirical determination of best practices and effective guidelines. In Proceedings of the ACM Conference on Designing Interactive Systems (DIS 2006), pp. 12-21, 2006.

27. von Oech, R. A whack on the side of the head. Hachette Book Group, New York, 2008.

28. Wahid, S. Branham, S. M., Cairco, L., McCrickard, D. S., Harrison, S. Picking Up Artifacts: Storyboarding as a Gateway to Reuse, In Proceedings of the IFIP TC.13 Conference on Human-Computer Interaction (INTERACT '09), pp 528-541, 2009.

29. Whittaker, S. Things to talk about when talking about things. Human-Computer Interaction, 18, pp. 149-170, 2003.

30. Yahoo! Patterns Library, http://developer.yahoo.com/ypattern 